\title{
10. Wenn Ihnen das alles noch nichts geholfen hat ...
}

Dieses Buch enthält eine sehr große Menge an Information, die Sie erst einmal verdauen und vor allem in der Praxis umsetzen müssen. Und: Es wird sicherlich Punkte geben, an denen Ihnen das hier vermittelte Grundwissen nicht ausreicht. Was tun? Hier können Sie immer auf das Fachwissen Ihrer Bibliothek zurückgreifen. Jede Hochschulbibliothek verfügt über hochqualifizierte Bibliothekarinnen und Bibliothekare, die zumeist ein einschlägiges Fachstudium absolviert und oft sogar promoviert, also selbst umfassend wissenschaftlich gearbeitet haben. Sie dienen als fachliche Ansprechpartner für die Studierenden und Lehrenden eines oder mehrerer Fächer - oft heißen sie Fachreferenten.

Diese fachlichen Ansprechpartner sind diejenigen, die sämtliche Bücher, die Sie lesen, und sämtliche elektronischen Medien, in denen Sie recherchieren, nach Qualitätskriterien ausgesucht haben. Sie kennen sich mit den Details Ihres Wissenschaftsfachs und den spezifischen Rechercheproblemen aus und können Ihnen in der weiten Welt der Recherchemedien meist auf Anhieb mehrere Schritte weiterhelfen. Über fachspezifische Schulungen und E-Learning-Angebote hinaus stehen diese Ansprechpartner Ihnen immer auch für ganz individuelle Beratungen zur Verfügung, beispielsweise, wenn Sie eine längere Abschlussarbeit schreiben. Nutzen Sie diesen Service! 